\title{
CONVERGENCE OF SOLUTIONS OF BILATERAL PROBLEMS IN VARIABLE DOMAINS AND RELATED QUESTIONS
}

\author{
Alexander A. Kovalevsky \\ Krasovskii Institute of Mathematics and Mechanics, \\ Ural Branch of Russian Academy of Sciences; \\ Ural Federal University, Ekaterinburg, Russia \\ alexkv171@mail.ru
}

\begin{abstract}
We discuss some results on the convergence of minimizers and minimum values of integral and more general functionals on sets of functions defined by bilateral constraints in variable domains. We consider the case of regular constraints, i.e., constraints lying in the corresponding Sobolev space, and the case where the lower constraint is zero and the upper constraint is an arbitrary nonnegative function. The first case concerns a larger class of integrands and requires the positivity almost everywhere of the difference between the upper and lower constraints. In the second case, this requirement is absent. Moreover, in the latter case, the exhaustion condition of an $n$-dimensional domain by a sequence of $n$-dimensional domains plays an important role. We give a series of results involving this condition. In particular, using the exhaustion condition, we prove a certain convergence of sets of functions defined by bilateral (generally irregular) constraints in variable domains.
\end{abstract}

Key words: Integral functional, Bilateral problem, Minimizer, Minimum value, $\Gamma$-convergence of functionals, Strong connectedness of spaces, $\mathcal{H}$-convergence of sets, Exhaustion condition.

\section{Introduction}

This paper is mainly based on the talk given by the author at the International S.B. Stechkin Summer Workshop-Conference on Function Theory, Miass, Russia, August 1-10, 2017.

The problems considered in the paper are related to the following general problem. Let $\left\{W_{s}\right\}$ be a sequence of Banach spaces, and let, for every $s \in \mathbb{N}, \mathcal{I}_{s}: W_{s} \rightarrow \mathbb{R}$ and $V_{s} \subset W_{s}, V_{s} \neq \varnothing$. Let, for every $s \in \mathbb{N}, u_{s}$ be a minimizer of $\mathcal{I}_{s}$ on $V_{s}$. The questions are, what are general conditions under which the sequence $\left\{u_{s}\right\}$ converges in a certain sense to an element and this limit element minimizes a functional $\mathcal{I}$ on a set $V$, and how are the functional $\mathcal{I}$ and the set $V$ related to the sequences $\left\{\mathcal{I}_{s}\right\}$ and $\left\{V_{s}\right\}$ ? Problems of this kind are studied in the framework of homogenization theory. There is a special kind of convergence of functionals that helps to solve the mentioned problems. This is the $\Gamma$-convergence. There are many works devoted to the study of this convergence. The $\Gamma$-convergence of functionals with the same domain of definition was studied, for instance, in [1-3]. In the simplest case, the definition of $\Gamma$-convergence is as follows.

Definition 1. Let, for every $s \in \mathbb{N}, f_{s}: \mathbb{R} \rightarrow \mathbb{R}$, and let $f: \mathbb{R} \rightarrow \mathbb{R}$. We say that the sequence $\left\{f_{s}\right\} \Gamma$-converges to the function $f$ if the following conditions are satisfied:

(a) for every $x \in \mathbb{R}$, there exists a sequence $\left\{y_{s}\right\} \subset \mathbb{R}$ such that $y_{s} \rightarrow x$ and $f_{s}\left(y_{s}\right) \rightarrow f(x)$;

(b) for every $x \in \mathbb{R}$ and every sequence $\left\{x_{s}\right\} \subset \mathbb{R}$ such that $x_{s} \rightarrow x$, we have the inequality $\liminf _{s \rightarrow \infty} f_{s}\left(x_{s}\right) \geqslant f(x)$.

The $\Gamma$-convergence of ordinary real functions and functionals defined on Banach spaces has some interesting properties that distinguish it from other kinds of convergence of the corresponding mappings. Among various properties of the $\Gamma$-convergence, we only mention its variational property that describes the relation of this convergence of functionals to the convergence of their minimizers 
and minimum values. A simple version of the variational property of the $\Gamma$-convergence is the following proposition.

Proposition 1. Let, for every $s \in \mathbb{N}, f_{s}: \mathbb{R} \rightarrow \mathbb{R}$, and let $f: \mathbb{R} \rightarrow \mathbb{R}$. Assume that the sequence $\left\{f_{s}\right\} \Gamma$-converges to the function $f$. Let, for every $s \in \mathbb{N}, x_{s}$ be a minimizer of $f_{s}$ on $\mathbb{R}$. Assume that $x_{s} \rightarrow x$. Then $x$ minimizes $f$ on $\mathbb{R}$ and $f_{s}\left(x_{s}\right) \rightarrow f(x)$.

$\mathrm{P}$ r o o f. Since $x_{s} \rightarrow x$, by condition (b) in Definition 1 , we have

$$
\liminf _{s \rightarrow \infty} f_{s}\left(x_{s}\right) \geqslant f(x) .
$$

Now, let $y \in \mathbb{R}$. By virtue of condition (a) in Definition 1 , there exists a sequence $\left\{y_{s}\right\} \subset \mathbb{R}$ such that

$$
f_{s}\left(y_{s}\right) \rightarrow f(y) .
$$

Since, for every $s \in \mathbb{N}, x_{s}$ minimizes $f_{s}$ on $\mathbb{R}$, we have

$$
\forall s \in \mathbb{N}, \quad f_{s}\left(x_{s}\right) \leqslant f_{s}\left(y_{s}\right) .
$$

Relations (2) and (3) imply that

$$
\limsup _{s \rightarrow \infty} f_{s}\left(x_{s}\right) \leqslant f(y) .
$$

From (1) and (4), we derive that $x$ minimizes $f$ on $\mathbb{R}$ and $f_{s}\left(x_{s}\right) \rightarrow f(x)$. We note that the latter limit relation follows from inequality (1) and from inequality (4) with $y=x$.

Here, we have restricted ourselves only to a simplest version of the variational property of the $\Gamma$-convergence, having shown how both conditions (a) and (b) in Definition 1 work. The considered case is very simple not only due the fact that we dealt with functions defined on $\mathbb{R}$ but also because of the assumption that the minimizers of these functions are global. In the case of minimizers on sets defined by certain constraints, the situation is more complicated, and not always the "global" $\Gamma$-convergence (i.e., the convergence of the kind described in Definition 1 with a $\Gamma$-realizing sequence $\left\{y_{s}\right\}$ taken in the whole corresponding space) can be used for the study of the convergence of such minimizers.

There are analogues of the above definition of $\Gamma$-convergence for functionals defined on a Banach space (in particular, on a Lebesgue or Sobolev space). In this connection, see, for instance, $[2,4]$. The notion of $\Gamma$-convergence of functionals with varying domain of definition (in particular, of functionals $\mathcal{I}_{s}: W^{m, p}\left(\Omega_{s}\right) \rightarrow \mathbb{R}$ with taking into account the structure of domains $\Omega_{s}$ ) was introduced and studied, for instance, in [5-7].

Next, note that, in the study of the convergence of minimizers $u_{s}$ of functionals $\mathcal{I}_{s}: W_{s} \rightarrow \mathbb{R}$, a connection of the spaces $W_{s}$ with a space $W$ plays an important role. Often, this connection is expressed as the requirement that there exists a sequence of operators $l_{s}: W_{s} \rightarrow W$ with certain properties. In particular, these properties should provide the following property: for every sequence $v_{s} \in W_{s}$ such that $\sup _{s \in \mathbb{N}}\left\|v_{s}\right\|_{W_{s}}<+\infty$, the sequence $\left\{l_{s} v_{s}\right\}$ is bounded in $W$. Under appropriate and in some sense natural conditions on the functionals $\mathcal{I}_{s}$, for the sequence of minimizers $u_{s} \in W_{s}$ of the functionals $\mathcal{I}_{s}$, the inequality $\sup _{s \in \mathbb{N}}\left\|u_{s}\right\|_{W_{s}}<+\infty$ holds. Therefore, if there exists a sequence $l_{s}: W_{s} \rightarrow W$ with the above mentioned property, then the sequence $\left\{l_{s} u_{s}\right\}$ is bounded. Consequently, if the space $W$ is reflexive, there exist an increasing sequence $\left\{s_{j}\right\} \subset \mathbb{N}$ and an element $u \in W$ such that $l_{s_{j}} u_{s_{j}} \rightarrow u$ weakly in $W$. Actually, this is the first step in the study of the convergence of the sequence of minimizers $u_{s} \in W_{s}$ of the functionals $\mathcal{I}_{s}$. The described idea with the operators $l_{s}$ is realized in the justification of the results stated below for functionals defined 
on the Sobolev spaces $W^{1, p}\left(\Omega_{s}\right)$, where $\left\{\Omega_{s}\right\}$ is a sequence of domains contained in a bounded domain $\Omega$ of $\mathbb{R}^{n}$. Essentially, the mentioned idea goes back to [8]. In this connection, see also [5-7].

The main content of this paper is organized as follows. In Section 1, we state the initial assumptions and the necessary definitions. In Section 2, we present our results on the convergence of minimizers and minimum values of integral and more general functionals on sets of functions defined by bilateral constraints in variable domains. We consider the case of regular constraints, i.e., constraints lying in the corresponding Sobolev space (see [9]), and the case where the lower constraint is zero and the upper constraint is an arbitrary nonnegative function (in this connection, see [10]). In both cases, a certain connection of the spaces $W^{1, p}\left(\Omega_{s}\right)$ with the space $W^{1, p}(\Omega)$ and the $\Gamma$-convergence of functionals defined on the spaces $W^{1, p}\left(\Omega_{s}\right)$ to a functional defined on $W^{1, p}(\Omega)$ are essentially used. At the same time, some other conditions on the involved domains, integrands, and constraints are also important for our convergence results. On the whole, the conditions providing these results are discussed in Section 3, where a special attention is paid to the so-called exhaustion condition of the domain $\Omega$ by the domains $\Omega_{s}$. This condition is the requirement that, for every increasing sequence $\left\{m_{j}\right\} \subset \mathbb{N}$, the measure of the union of all the domains $\Omega_{m_{j}}$ is equal to the measure of the domain $\Omega$. We also consider the notion of $\mathcal{H}$-convergence of sequences of sets $U_{s} \subset W^{1, p}\left(\Omega_{s}\right)$ to a set $U \subset W^{1, p}(\Omega)$ and show the importance of the exhaustion condition for the $\mathcal{H}$-convergence of sets of functions defined by irregular bilateral constraints.

\section{Assumptions and definitions}

Let $n \in \mathbb{N}, n \geqslant 2$, let $\Omega$ be a bounded domain of $\mathbb{R}^{n}$, and let $p>1$. Let $\left\{\Omega_{s}\right\}$ be a sequence of domains of $\mathbb{R}^{n}$ contained in $\Omega$.

It is easy to see that if $v \in W^{1, p}(\Omega)$ and $s \in \mathbb{N}$, then $\left.v\right|_{\Omega_{s}} \in W^{1, p}\left(\Omega_{s}\right)$.

Definition 2. If $s \in \mathbb{N}$, then $q_{s}: W^{1, p}(\Omega) \rightarrow W^{1, p}\left(\Omega_{s}\right)$ is the mapping such that, for every function $v \in W^{1, p}(\Omega)$, we have $q_{s} v=\left.v\right|_{\Omega_{s}}$.

Definition 3. We say that the sequence of spaces $W^{1, p}\left(\Omega_{s}\right)$ is strongly connected with the space $W^{1, p}(\Omega)$ if there exists a sequence of linear continuous operators $l_{s}: W^{1, p}\left(\Omega_{s}\right) \rightarrow W^{1, p}(\Omega)$ such that:

(a) the sequence of norms $\left\|l_{s}\right\|$ is bounded;

(b) for every $s \in \mathbb{N}$ and for every $v \in W^{1, p}\left(\Omega_{s}\right)$, we have $q_{s}\left(l_{s} v\right)=v$ a.e. in $\Omega_{s}$.

The prototype of the notion in Definition 3 is the condition of strong connectedness of $n$-dimensional domains introduced in [8].

Definition 4. Let, for every $s \in \mathbb{N}, I_{s}: W^{1, p}\left(\Omega_{s}\right) \rightarrow \mathbb{R}$, and let $I: W^{1, p}(\Omega) \rightarrow \mathbb{R}$. We say that the sequence $\left\{I_{s}\right\} \quad \Gamma$-converges to the functional $I$ if the following conditions are satisfied:

(a) for every function $v \in W^{1, p}(\Omega)$, there exists a sequence $w_{s} \in W^{1, p}\left(\Omega_{s}\right)$ such that $\left\|w_{s}-q_{s} v\right\|_{L^{p}\left(\Omega_{s}\right)} \rightarrow 0$ and $I_{s}\left(w_{s}\right) \rightarrow I(v)$

(b) for every function $v \in W^{1, p}(\Omega)$ and for every sequence $v_{s} \in W^{1, p}\left(\Omega_{s}\right)$ such that $\left\|v_{s}-q_{s} v\right\|_{L^{p}\left(\Omega_{s}\right)} \rightarrow 0$, we have $\liminf _{s \rightarrow \infty} I_{s}\left(v_{s}\right) \geqslant I(v)$.

Next, let $c_{1}, c_{2}>0$, and let, for every $s \in \mathbb{N}, \mu_{s} \in L^{1}\left(\Omega_{s}\right)$ and $\mu_{s} \geqslant 0$ in $\Omega_{s}$. We assume that the sequence of norms $\left\|\mu_{s}\right\|_{L^{1}\left(\Omega_{s}\right)}$ is bounded.

Let, for every $s \in \mathbb{N}, f_{s}: \Omega_{s} \times \mathbb{R}^{n} \rightarrow \mathbb{R}$ be a function satisfying the following conditions: for every $\xi \in \mathbb{R}^{n}$, the function $f_{s}(\cdot, \xi)$ is measurable on $\Omega_{s}$; for almost every $x \in \Omega_{s}$, the function $f_{s}(x, \cdot)$ is convex on $\mathbb{R}^{n}$; for almost every $x \in \Omega_{s}$ and for every $\xi \in \mathbb{R}^{n}$, we have

$$
c_{1}|\xi|^{p}-\mu_{s}(x) \leqslant f_{s}(x, \xi) \leqslant c_{2}|\xi|^{p}+\mu_{s}(x) .
$$


In view of the assumptions on the functions $f_{s}$ and $\mu_{s}$, for every $s \in \mathbb{N}$ and for every $v \in W^{1, p}\left(\Omega_{s}\right)$, the function $f_{s}(x, \nabla v)$ is summable on $\Omega_{s}$.

Definition 5. If $s \in \mathbb{N}$, then $F_{s}: W^{1, p}\left(\Omega_{s}\right) \rightarrow \mathbb{R}$ is the functional such that, for every function $v \in W^{1, p}\left(\Omega_{s}\right)$, we have

$$
F_{s}(v)=\int_{\Omega_{s}} f_{s}(x, \nabla v) d x .
$$

By virtue of the conditions on the functions $f_{s}$, for every $s \in \mathbb{N}$, the functional $F_{s}$ is convex and locally bounded. Therefore, for every $s \in \mathbb{N}$, the functional $F_{s}$ is weakly lower semicontinuous.

Let $c_{3}, c_{4}>0$, and let, for every $s \in \mathbb{N}, G_{s}: W^{1, p}\left(\Omega_{s}\right) \rightarrow \mathbb{R}$ be a weakly continuous functional. We assume that, for every $s \in \mathbb{N}$ and for every $v \in W^{1, p}\left(\Omega_{s}\right)$,

$$
G_{s}(v) \geqslant c_{3}\|v\|_{L^{p}\left(\Omega_{s}\right)}^{p}-c_{4} .
$$

Obviously, for every $s \in \mathbb{N}$, the functional $F_{s}+G_{s}$ is weakly lower semicontinuous. Moreover, in view of (5) and (6) and the boundedness of the sequence of norms $\left\|\mu_{s}\right\|_{L^{1}\left(\Omega_{s}\right)}$, there exist positive constants $c_{5}$ and $c_{6}$ such that, for every $s \in \mathbb{N}$ and for every $v \in W^{1, p}\left(\Omega_{s}\right)$, we have

$$
\left(F_{s}+G_{s}\right)(v) \geqslant c_{5}\|v\|_{W^{1, p}\left(\Omega_{s}\right)}^{p}-c_{6} .
$$

Thus, in view of the known results on the existence of minimizers of functionals (see, for instance, [11]), if $s \in \mathbb{N}$ and $U_{s}$ is a sequentially weakly closed set in $W^{1, p}\left(\Omega_{s}\right)$, then there exists a minimizer of the functional $F_{s}+G_{s}$ on the set $U_{s}$.

\section{Variational problems with bilateral constraints}

First, we consider the case of regular bilateral constraints.

Let $\varphi, \psi \in W^{1, p}(\Omega)$, and let $\varphi \leqslant \psi$ a.e. in $\Omega$. We define

$$
V(\varphi, \psi)=\left\{v \in W^{1, p}(\Omega): \varphi \leqslant v \leqslant \psi \text { a.e. in } \Omega\right\},
$$

and let, for every $s \in \mathbb{N}$,

$$
V_{s}(\varphi, \psi)=\left\{v \in W^{1, p}\left(\Omega_{s}\right): \varphi \leqslant v \leqslant \psi \text { a.e. in } \Omega_{s}\right\} .
$$

It is easy to see that the set $V(\varphi, \psi)$ is nonempty, closed, and convex. Similarly, for every $s \in \mathbb{N}$, the set $V_{s}(\varphi, \psi)$ is nonempty, closed, and convex.

Clearly, for every $s \in \mathbb{N}$, there exists a function belonging to the set $V_{s}(\varphi, \psi)$ and minimizing the functional $F_{s}+G_{s}$ on this set.

Theorem 1. Assume that the following conditions are satisfied:

$\left(*_{1}\right)$ the embedding of $W^{1, p}(\Omega)$ into $L^{p}(\Omega)$ is compact;

$\left(*_{2}\right)$ the sequence of spaces $W^{1, p}\left(\Omega_{s}\right)$ is strongly connected with the space $W^{1, p}(\Omega)$;

$\left(*_{3}\right)$ for every sequence of measurable sets $H_{s} \subset \Omega_{s}$ such that meas $H_{s} \rightarrow 0$, we have

$$
\int_{H_{s}} \mu_{s} d x \rightarrow 0
$$

$\left(*_{4}\right)$ the sequence $\left\{F_{s}\right\} \Gamma$-converges to a functional $F: W^{1, p}(\Omega) \rightarrow \mathbb{R}$;

$\left(*_{5}\right)$ there exists a functional $G: W^{1, p}(\Omega) \rightarrow \mathbb{R}$ such that, for every function $v \in W^{1, p}(\Omega)$ and for every sequence $v_{s} \in W^{1, p}\left(\Omega_{s}\right)$ with the property $\left\|v_{s}-q_{s} v\right\|_{L^{p}\left(\Omega_{s}\right)} \rightarrow 0$, we have $G_{s}\left(v_{s}\right) \rightarrow G(v)$; 
$\left(*_{6}\right) \psi-\varphi>0$ a.e. in $\Omega$.

Let, for every $s \in \mathbb{N}, u_{s}$ be a function in $V_{s}(\phi, \psi)$ minimizing the functional $F_{s}+G_{s}$ on the set $V_{s}(\varphi, \psi)$. Then there exist an increasing sequence $\left\{s_{j}\right\} \subset \mathbb{N}$ and a function $u \in V(\varphi, \psi)$ such that $u$ minimizes the functional $F+G$ on the set $V(\varphi, \psi),\left\|u_{s_{j}}-q_{s_{j}} u\right\|_{L^{p}\left(\Omega_{s_{j}}\right)} \rightarrow 0$, and $\left(F_{s_{j}}+G_{s_{j}}\right)\left(u_{s_{j}}\right) \rightarrow(F+G)(u)$.

Essentially, a similar result was obtained in [12] but under stronger assumptions on the functionals $F_{s}$ and $G_{s}$ and under the condition $\psi-\varphi \geqslant \alpha$ a.e. in $\Omega$, where $\alpha>0$. In this connection, see also [13, Theorem 2.9].

Concerning the proof of Theorem 1, we note the following. First, using operators $l_{s}: W^{1, p}\left(\Omega_{s}\right) \rightarrow$ $W^{1, p}(\Omega)$ described in Definition 3 and defining the functions $\tilde{u}_{s}=\min \left\{\max \left\{l_{s} u_{s}, \varphi\right\}, \psi\right\}$, we find that there exist an increasing sequence $\left\{s_{j}\right\} \subset \mathbb{N}$ and a function $u \in W^{1, p}(\Omega)$ such that $\tilde{u}_{s_{j}} \rightarrow u$ strongly in $L^{p}(\Omega)$ and almost everywhere in $\Omega$. Then we obtain the inclusion $u \in V(\varphi, \psi)$, the limit relation $\left\|u_{s_{j}}-q_{s_{j}} u\right\|_{L^{p}\left(\Omega_{s_{j}}\right)} \rightarrow 0$, and, by virtue of conditions $\left(*_{4}\right)$ and $\left(*_{5}\right)$ of Theorem 1 , the inequality $\liminf _{s \rightarrow \infty}\left(F_{s_{j}}+G_{s_{j}}\right)\left(u_{s_{j}}\right) \geqslant(F+G)(u)$. The next and most important step is to establish, for every function $v \in V(\varphi, \psi)$, the existence of a sequence $w_{s} \in V_{s}(\varphi, \psi)$ with the following properties: $\left\|w_{s}-q_{s} v\right\|_{L^{p}\left(\Omega_{s}\right)} \rightarrow 0$ and

$$
\limsup _{s \rightarrow \infty} F_{s}\left(w_{s}\right) \leqslant F(v) .
$$

The construction of such a sequence involves the function $v$ and a $\Gamma$-realizing sequence $\left\{v_{s}\right\}$ for $v$, i.e., a sequence $v_{s} \in W^{1, p}\left(\Omega_{s}\right)$ such that $\left\|v_{s}-q_{s} v\right\|_{L^{p}\left(\Omega_{s}\right)} \rightarrow 0$ and $F_{s}\left(v_{s}\right) \rightarrow F(v)$, which exists in view of condition $\left(*_{4}\right)$ of Theorem 1 . Moreover, it involves the difference $\psi-\varphi$. Using the limit relation $\left\|v_{s}-q_{s} v\right\|_{L^{p}\left(\Omega_{s}\right)} \rightarrow 0$ and condition $\left(*_{6}\right)$ of Theorem 1, we find that, for a sequence $\left\{\sigma_{s}\right\} \subset(0,1]$ converging to 0 , meas $\left\{\left|v_{s}-q_{s} v\right| \geqslant \sigma_{s} q_{s}(\psi-\varphi)\right\} \rightarrow 0$. This is a key moment in the proof of inequality (8). For further details leading to the required properties of the function $u$, see $[9$, Section 2].

We now proceed to the case of irregular bilateral constraints. More precisely, we consider the case where the lower constraint is zero and the upper constraint is an arbitrary nonnegative function. Thus, in contrast to the previous case, the upper constraint can be irregular and both constraints can coincide on a set of positive measure. This is due to an additional condition on the domains $\Omega_{s}$ and a stronger condition on the functions $\mu_{s}$ as compared to condition $\left(*_{3}\right)$ of Theorem 1.

Let $\psi: \Omega \rightarrow \overline{\mathbb{R}}$ and $\psi \geqslant 0$ a.e. in $\Omega$. We define

$$
V(\psi)=\left\{v \in W^{1, p}(\Omega): 0 \leqslant v \leqslant \psi \text { a.e. in } \Omega\right\},
$$

and let, for every $s \in \mathbb{N}$,

$$
V_{s}(\psi)=\left\{v \in W^{1, p}\left(\Omega_{s}\right): 0 \leqslant v \leqslant \psi \text { a.e. in } \Omega_{s}\right\} .
$$

It is easy to see that the set $V(\psi)$ is nonempty, closed, and convex. Moreover, for every $s \in \mathbb{N}$, the set $V_{s}(\psi)$ is nonempty, closed, and convex.

Obviously, for every $s \in \mathbb{N}$, there exists a function belonging to the set $V_{s}(\psi)$ and minimizing the functional $F_{s}+G_{s}$ on this set.

Theorem 2. Assume that conditions $\left(*_{1}\right),\left(*_{2}\right),\left(*_{4}\right)$, and $\left(*_{5}\right)$ of Theorem 1 are satisfied. In addition, suppose that the following conditions are satisfied:

$\left(*^{\prime}\right)$ for every increasing sequence $\left\{m_{j}\right\} \subset \mathbb{N}$, we have meas $\left(\Omega \backslash \bigcup_{j=1}^{\infty} \Omega_{m_{j}}\right)=0$; 
$\left(*^{\prime \prime}\right)\left\|\mu_{s}\right\|_{L^{1}\left(\Omega_{s}\right)} \rightarrow 0$

Let, for every $s \in \mathbb{N}$, $u_{s}$ be a function in $V_{s}(\psi)$ minimizing the functional $F_{s}+G_{s}$ on the set $V_{s}(\psi)$. Then there exist an increasing sequence $\left\{s_{j}\right\} \subset \mathbb{N}$ and a function $u \in V(\psi)$ such that $u$ minimizes the functional $F+G$ on the set $V(\psi),\left\|u_{s_{j}}-q_{s_{j}} u\right\|_{L^{p}\left(\Omega_{s_{j}}\right)} \rightarrow 0$, and $\left(F_{s_{j}}+G_{s_{j}}\right)\left(u_{s_{j}}\right) \rightarrow$ $(F+G)(u)$.

As for the proof of Theorem 2, we give the following remarks. Since, in general, the function $\psi$ is irregular, we cannot use functions like the above functions $\tilde{u}_{s}$ in the proof of Theorem 1 . Therefore, using operators $l_{s}: W^{1, p}\left(\Omega_{s}\right) \rightarrow W^{1, p}(\Omega)$ described in Definition 3, first, we find that there exist an increasing sequence $\left\{s_{j}\right\} \subset \mathbb{N}$ and a function $u \in W^{1, p}(\Omega)$ such that $l_{s_{j}} u_{s_{j}} \rightarrow u$ strongly in $L^{p}(\Omega)$ and almost everywhere in $\Omega$. Then, to prove that $u \in V(\psi)$, along with the inclusions $u_{s} \in V_{s}(\psi)$, we use condition $\left(*^{\prime}\right)$ of Theorem 2 which effectively works in this situation. Similarly to the proof of Theorem 1, the most important step in the proof of Theorem 2 is to establish, for every function $v \in V(\psi)$, the existence of a sequence $w_{s} \in V_{s}(\psi)$ such that $\left\|w_{s}-q_{s} v\right\|_{L^{p}\left(\Omega_{s}\right)} \rightarrow 0$ and inequality (8) holds. The construction of such a sequence involves the function $v$ and a $\Gamma$-realizing sequence $\left\{v_{s}\right\}$ for $v$ but does not involve the constraint $\psi$. To prove inequality (8), we essentially use condition $\left(*^{\prime \prime}\right)$ of Theorem 2 and the fact that meas $\left(\left\{\left|v_{s}-q_{s} v\right| \geqslant \sigma_{s} q_{s} v\right\} \cap\{v>0\}\right) \rightarrow 0$, where $\left\{\sigma_{s}\right\}$ is a sequence in $[0,1)$ such that $\sigma_{s} \rightarrow 0$. For details, see the proof of Theorem 3.1 in [10].

The next result describes a situation where we have the convergence of the whole sequence of minimizers and of the whole sequence of minimum values.

Theorem 3. Assume that conditions $\left(*_{1}\right),\left(*_{2}\right),\left(*_{4}\right)$, and $\left(*_{5}\right)$ of Theorem 1 are satisfied, and the functional $G$ is strictly convex on the set $V(\psi)$. In addition, suppose that conditions ( $*^{\prime}$ ) and $\left(*^{\prime \prime}\right)$ of Theorem 2 are satisfied. Let, for every $s \in \mathbb{N}, u_{s}$ be a function in $V_{s}(\psi)$ minimizing the functional $F_{s}+G_{s}$ on the set $V_{s}(\psi)$. Then there exists a unique function $u \in V(\psi)$ minimizing the functional $F+G$ on the set $V(\psi)$ and the following relations hold: $\left\|u_{s}-q_{s} u\right\|_{L^{p}\left(\Omega_{s}\right)} \rightarrow 0$ and $\left(F_{s}+G_{s}\right)\left(u_{s}\right) \rightarrow(F+G)(u)$.

\section{Comments to the conditions of Theorems 1-3}

As is known (see, for instance, [14, Chapter 6]), condition $\left(*_{1}\right)$ of Theorem 1 is satisfied if $\Omega$ is a Lipschitz domain. In particular, bounded convex domains are Lipschitz domains. A more general requirement guaranteeing the fulfillment of condition $\left(*_{1}\right)$ is that $\Omega$ is an extension domain (see, for instance, [15, Chapter 1]).

Condition $\left(*_{2}\right)$ of Theorem 1 is satisfied, in particular, if the domains $\Omega_{s}$ have a certain perforated structure. In this regard, see, for instance, [16, Section 2].

As far as conditions $\left(*_{3}\right)$ and $\left(*_{4}\right)$ of Theorem 1 are concerned, we note the following. In the case where the functions $\mu_{s}$ take a constant value independent of $s$, theorems on conditions for the $\Gamma$-convergence of the integral functionals $F_{s}$ with the integrands $f_{s}$ satisfying condition $(5)$ follow from the results of $[17,18]$, where the $\Gamma$-convergence of integral functionals defined on the spaces $W^{m, p}\left(\Omega_{s}\right)$ with an arbitrary $m \in \mathbb{N}$ was studied. In this case, the sequence $\left\{F_{s}\right\} \Gamma$-converges to an integral functional defined on the space $W^{1, p}(\Omega)$, in particular, if the domains $\Omega_{s}$ have a periodic perforated structure and all the integrands $f_{s}$ coincide with the same integrand having a certain regularity (see [17]). Obviously, in the specified case for the functions $\mu_{s}$, the sequence of norms $\left\|\mu_{s}\right\|_{L^{1}\left(\Omega_{s}\right)}$ is bounded and condition $\left(*_{3}\right)$ of Theorem 1 is satisfied. In the more general case where $\mu_{s} \in L^{1}\left(\Omega_{s}\right)$ and $\mu_{s} \geqslant 0$ in $\Omega_{s}$ for every $s \in \mathbb{N}$ and, in addition, the inequality

$$
\limsup _{s \rightarrow \infty} \int_{Q \cap \Omega_{s}} \mu_{s} d x \leqslant \int_{Q \cap \Omega} \mu d x
$$


holds for a function $\mu \in L^{1}(\Omega), \mu \geqslant 0$ in $\Omega$, and for every open cube $Q$ of $\mathbb{R}^{n}$, a theorem on the $\Gamma$-compactness of the sequence $\left\{F_{s}\right\}$ can be proved similarly to the corresponding results in [19, 20]. Obviously, in this case, the sequence of norms $\left\|\mu_{s}\right\|_{L^{1}\left(\Omega_{s}\right)}$ is bounded. We also note that there are examples of sequences of nonnegative functions $\mu_{s} \in L^{1}\left(\Omega_{s}\right)$ for which condition (9) and condition $\left(*_{3}\right)$ of Theorem 1 are satisfied but there is no function $\mu_{*}: \Omega \rightarrow \mathbb{R}$ such that, for every $s \in \mathbb{N}, \mu_{s} \leqslant \mu_{*}$ a.e. in $\Omega_{s}$. Such examples can be given with the use of the functions constructed in $[21]$.

In connection with condition $\left(*_{5}\right)$ of Theorem 1 , we give the following example.

Example 1. Let $a \in L^{p /(p-1)}(\Omega)$. Let $\beta_{1} \in(0,1)$, let $\beta_{2}>0$, and let $\Phi:[0,+\infty) \rightarrow \mathbb{R}$ be a continuous function such that

$$
\forall \eta \in[0,+\infty), \quad|\Phi(\eta)| \leqslant \beta_{1}|\eta|^{p}+\beta_{2} .
$$

For every $s \in \mathbb{N}$, we define the functional $G_{s}: W^{1, p}\left(\Omega_{s}\right) \rightarrow \mathbb{R}$ by

$$
G_{s}(v)=\int_{\Omega_{s}}\left\{|v|^{p}+a v\right\} d x+\Phi\left(\|v\|_{L^{p}\left(\Omega_{s}\right)}\right), \quad v \in W^{1, p}\left(\Omega_{s}\right) .
$$

In view of (10), for every $s \in \mathbb{N}$ and for every $v \in W^{1, p}\left(\Omega_{s}\right)$, inequality (6) holds with constants $c_{3}$ and $c_{4}$ depending only on $p, \beta_{1}, \beta_{2}$, and $\|a\|_{L^{p /(p-1)}(\Omega)}$. We also note that if conditions $\left(*_{1}\right)$ and $\left(*_{2}\right)$ of Theorem 1 are satisfied, then, for every $s \in \mathbb{N}$, the functional $G_{s}$ is weakly continuous. Next, assume that the following condition is satisfied:

$(*)$ there exists a nonnegative bounded measurable function $b: \Omega \rightarrow \mathbb{R}$ such that, for every open cube $Q \subset \Omega$, we have meas $\left(Q \cap \Omega_{s}\right) \rightarrow \int_{Q} b d x$.

Now, let $G: W^{1, p}(\Omega) \rightarrow \mathbb{R}$ be the functional such that, for every function $v \in W^{1, p}(\Omega)$, we have

$$
G(v)=\int_{\Omega} b\left\{|v|^{p}+a v\right\} d x+\Phi\left(\left\|b^{1 / p} v\right\|_{L^{p}(\Omega)}\right) .
$$

Using condition $(*)$ and the continuity of the function $\Phi$, we find that, for the sequence of functionals $G_{s}$, condition $\left(*_{5}\right)$ of Theorem 1 is satisfied.

We remark that if the domain $\Omega$ is Lipschitz and the domains $\Omega_{s}$ have a certain periodically perforated structure, then conditions $\left(*_{1}\right)$ and $\left(*_{2}\right)$ of Theorem 1 are satisfied along with condition $(*)$ in which the function $b$ takes a constant positive value. Obviously, for such a function $b$, the functional $G$ defined by (11) is strictly convex if the function $\Phi$ is nondecreasing and convex.

We emphasize the importance of condition $\left(*_{6}\right)$ of Theorem 1 for its conclusion. In [9], we gave an example where all the conditions of Theorem 1 are satisfied except for condition $\left(*_{6}\right)$ but the conclusion of this theorem does not hold on the whole. We note that, in this example, for an arbitrary pre-assigned positive $\varepsilon$, the measure of the set where the lower and upper constraints coincide does not exceed $\varepsilon$. Here is a simple example where condition $\left(*_{6}\right)$ of Theorem 1 is satisfied.

Example 2. Let $\Omega=\left\{x \in \mathbb{R}^{n}:|x|<1\right\}$, and let, for every $x \in \Omega$, we have $\varphi(x)=0$ and $\psi(x)=|x|^{2}\left(1-|x|^{2}\right)$. In view of these assumptions, we have $\varphi, \psi \in \stackrel{\circ}{W^{1, p}}(\Omega)$ and $\varphi \leqslant \psi$ in $\Omega$. In addition, for every $x \in \Omega \backslash\{0\},(\psi-\varphi)(x)>0$. Thus, condition $\left(*_{6}\right)$ of Theorem 1 is satisfied. We observe that, in the case considered here, we have $V(\varphi, \psi)=\left\{v \in \stackrel{\circ}{W}^{1, p}(\Omega): \varphi \leqslant v \leqslant \psi\right.$ a.e. in $\left.\Omega\right\}$. Hence, for $p=2$, the set $V(\varphi, \psi)$ has the same form as the set defined by bilateral constraints 
in [22]. We also note that if $\omega$ is a domain of $\mathbb{R}^{n}$ such that $\bar{\omega} \subset \Omega$ and the origin is contained in $\omega$, then there is no number $\delta^{\omega}>0$ such that $\psi-\varphi \geqslant \delta^{\omega}$ a.e. in $\omega$. We remark in this connection that it was shown in [22] that the $G$-convergence of a sequence of linear continuous divergence operators $A_{s}: \stackrel{\circ}{W}^{1,2}(\Omega) \rightarrow W^{-1,2}(\Omega)$ to an operator $A: \stackrel{\circ}{W}{ }^{1,2}(\Omega) \rightarrow W^{-1,2}(\Omega)$ of the same form implies the weak convergence of solutions of variational inequalities with the operators $A_{s}$ and the set of constraints $K\left(\psi_{1}, \psi_{2}\right)=\left\{v \in \stackrel{\circ}{W}^{1,2}(\Omega): \psi_{1} \leqslant v \leqslant \psi_{2}\right.$ a.e. in $\left.\Omega\right\}$ to the solution of the corresponding variational inequality with the operator $A$ and the same set of constraints. At the same time, it was assumed in [22] that $\psi_{1}, \psi_{2} \in L^{2}(\Omega)$ and, for every subdomain $\omega \subset \subset \Omega$, there exist a number $\delta^{\omega}>0$ and functions $\psi_{1}^{\omega}, \psi_{2}^{\omega} \in \stackrel{\circ}{W^{1,2}}(\Omega)$ such that $\psi_{1} \leqslant \psi_{1}^{\omega} \leqslant \psi_{2}^{\omega} \leqslant \psi_{2}$ in $\Omega$ and $\psi_{2}^{\omega}-\psi_{1}^{\omega} \geqslant \delta^{\omega}$ in $\omega$. Obviously, the functions $\varphi$ and $\psi$ defined at the beginning of this example do not satisfy the assumption given in [22].

We now discuss condition $\left(*^{\prime}\right)$ of Theorem 2. This condition is essential for the conclusion of Theorem 2. In [10], we construct an example where all the conditions of Theorem 2 are satisfied except for condition $\left(*^{\prime}\right)$ but the conclusion of this theorem does not hold. We call condition $\left(*^{\prime}\right)$ of Theorem 2 the exhaustion condition of the domain $\Omega$ by the domains $\Omega_{s}$. This condition plays an important role in the study of the convergence of solutions of variational problems with irregular unilateral and bilateral constraints in variable domains. In this regard, in addition to the present paper, see [23, 24]. We used the same exhaustion condition earlier in [6] for the investigation of both a convergence of sets in variable Sobolev spaces and the coercivity of the $\Gamma$-limit of functionals defined on these spaces. Below, we show how such questions are solved for sequences of sets $U_{s} \subset W^{1, p}\left(\Omega_{s}\right)$ and the functionals $F_{s}+G_{s}$. Before we do this, let us give some useful results.

Proposition 2. Condition $\left(*^{\prime}\right)$ of Theorem 2 is equivalent to the following condition:

$$
\text { if } v \in L^{1}(\Omega) \text { and } \liminf _{s \rightarrow \infty} \int_{\Omega_{s}}|v| d x=0 \text {, then } v=0 \text { a.e. in } \Omega \text {. }
$$

P r o o f. Assume that condition $\left(*^{\prime}\right)$ of Theorem 2 is satisfied. Let $v \in L^{1}(\Omega)$, and let

$$
\liminf _{s \rightarrow \infty} \int_{\Omega_{s}}|v| d x=0
$$

Fixing an arbitrary $\varepsilon>0$, we find that there exists an increasing sequence $\left\{s_{j}\right\} \subset \mathbb{N}$ such that

$$
\forall j \in \mathbb{N}, \quad \int_{\Omega_{s_{j}}}|v| d x \leqslant \frac{\varepsilon}{2^{j}} .
$$

Setting $\Omega^{\prime}=\bigcup_{j=1}^{\infty} \Omega_{s_{j}}$, by condition $\left(*^{\prime}\right)$ of Theorem 2 , we have meas $\left(\Omega \backslash \Omega^{\prime}\right)=0$. Then

$$
\int_{\Omega}|v| d x=\int_{\Omega^{\prime}}|v| d x \leqslant \sum_{j=1}^{\infty} \int_{\Omega_{s_{j}}}|v| d x .
$$

This and (13) imply that

$$
\int_{\Omega}|v| d x \leqslant \varepsilon
$$


Hence, in view of the arbitrariness of $\varepsilon$, we conclude that $v=0$ a.e. in $\Omega$. Thus, condition (12) is satisfied.

Conversely, assume that condition (12) is satisfied. Let $\left\{m_{j}\right\}$ be an increasing sequence in $\mathbb{N}$. Setting $E_{0}=\Omega \backslash \bigcup_{j=1}^{\infty} \Omega_{m_{j}}$, we suppose that meas $E_{0}>0$. Let $\chi: \Omega \rightarrow \mathbb{R}$ be the characteristic function of the set $E_{0}$. Obviously, $\chi \in L^{1}(\Omega)$ and $\int_{\Omega_{m_{j}}} \chi d x=0$ for every $j \in \mathbb{N}$. Therefore,

$$
\liminf _{s \rightarrow \infty} \int_{\Omega_{s}} \chi d x=0
$$

Then, by condition (12), we have $\chi=0$ a.e. in $\Omega$. Hence, there exists a set $E \subset \Omega$ of measure zero such that, for every $x \in \Omega \backslash E$, we have $\chi(x)=0$. Then, fixing $x \in E_{0} \backslash E$, we obtain $\chi(x)=0$. On the other hand, by the definition of the function $\chi$, we have $\chi(x)=1$. The obtained contradiction proves that meas $E_{0}=0$. Thus, condition $\left(*^{\prime}\right)$ of Theorem 2 is satisfied.

Proposition 3. Let condition $\left(*^{\prime}\right)$ of Theorem 2 be satisfied. Then the following condition is satisfied:

$$
\text { if } v \in W^{1, p}(\Omega) \text { and } \liminf _{s \rightarrow \infty}\left\|q_{s} v\right\|_{L^{p}\left(\Omega_{s}\right)}=0 \text {, then } v=0 \text { a.e. in } \Omega \text {. }
$$

P r o o f. Let $v \in W^{1, p}(\Omega)$ and $\liminf _{s \rightarrow \infty}\left\|q_{s} v\right\|_{L^{p}\left(\Omega_{s}\right)}=0$. Setting $w=|v|^{p}$, we have

$$
w \in L^{1}(\Omega), \quad \liminf _{s \rightarrow \infty} \int_{\Omega_{s}} w d x=0 .
$$

Since, by assumption, condition $\left(*^{\prime}\right)$ of Theorem 2 is satisfied, we deduce from Proposition 2 that condition (12) is satisfied. The latter condition along with (15) implies that $w=0$ a.e. in $\Omega$. Hence, $v=0$ a.e. in $\Omega$. Thus, condition (14) is satisfied.

Proposition 4. Let condition $\left(*_{1}\right)$ of Theorem 1 be satisfied, and assume that there exists a sequence of linear continuous operators $l_{s}: W^{1, p}\left(\Omega_{s}\right) \rightarrow W^{1, p}(\Omega)$ such that the sequence of norms $\left\|l_{s}\right\|$ is bounded and, for every $s \in \mathbb{N}$ and for every $v \in W^{1, p}\left(\Omega_{s}\right)$, we have $q_{s}\left(l_{s} v\right)=v$ a.e. in $\Omega_{s}$. Let, for every $s \in \mathbb{N}, w_{s} \in W^{1, p}\left(\Omega_{s}\right)$. Assume that the sequence of norms $\left\|w_{s}\right\|_{W^{1, p}\left(\Omega_{s}\right)}$ is bounded. Then there exist an increasing sequence $\left\{s_{j}\right\} \subset \mathbb{N}$ and a function $w \in W^{1, p}(\Omega)$ such that $l_{s_{j}} w_{s_{j}} \rightarrow w$ weakly in $W^{1, p}(\Omega), l_{s_{j}} w_{s_{j}} \rightarrow w$ a.e. in $\Omega$, and $\left\|w_{s_{j}}-q_{s_{j}} w\right\|_{L^{p}\left(\Omega_{s_{j}}\right)} \rightarrow 0$.

P r o o f. The properties of the operators $l_{s}$ along with the boundedness of the sequence of norms $\left\|w_{s}\right\|_{W^{1, p}\left(\Omega_{s}\right)}$ imply that the sequence $\left\{l_{s} w_{s}\right\}$ is bounded in $W^{1, p}(\Omega)$ and

$$
\forall s \in \mathbb{N}, \quad q_{s}\left(l_{s} w_{s}\right)=w_{s} \text { a.e. in } \Omega_{s} .
$$

Since the space $W^{1, p}(\Omega)$ is reflexive and the sequence $\left\{l_{s} w_{s}\right\}$ is bounded in $W^{1, p}(\Omega)$, there exist an increasing sequence $\left\{\bar{s}_{k}\right\} \subset \mathbb{N}$ and a function $w \in W^{1, p}(\Omega)$ such that $l_{\bar{s}_{k}} w_{\bar{s}_{k}} \rightarrow w$ weakly in $W^{1, p}(\Omega)$. Hence, by condition $\left(*_{1}\right)$ of Theorem 1 , we have $l_{\bar{s}_{k}} w_{\bar{s}_{k}} \rightarrow w$ strongly in $L^{p}(\Omega)$. Therefore, there exists an increasing sequence $\left\{s_{j}\right\} \subset\left\{\bar{s}_{k}\right\}$ such that $l_{s_{j}} w_{s_{j}} \rightarrow w$ a.e. in $\Omega$. It is clear that $l_{s_{j}} w_{s_{j}} \rightarrow w$ weakly in $W^{1, p}(\Omega)$ and $l_{s_{j}} w_{s_{j}} \rightarrow w$ strongly in $L^{p}(\Omega)$. The latter convergence along with (16) implies that $\left\|w_{s_{j}}-q_{s_{j}} w\right\|_{L^{p}\left(\Omega_{s_{j}}\right)} \rightarrow 0$. 
Proposition 5. Let condition $\left(*_{1}\right)$ of Theorem 1 be satisfied, and assume that there exists a sequence of linear continuous operators $l_{s}: W^{1, p}\left(\Omega_{s}\right) \rightarrow W^{1, p}(\Omega)$ such that the sequence of norms $\left\|l_{s}\right\|$ is bounded and, for every $s \in \mathbb{N}$ and for every $v \in W^{1, p}\left(\Omega_{s}\right)$, we have $q_{s}\left(l_{s} v\right)=v$ a.e. in $\Omega_{s}$. In addition, assume that condition $\left(*^{\prime}\right)$ of Theorem 2 is satisfied. Let, for every $s \in \mathbb{N}$, $w_{s} \in W^{1, p}\left(\Omega_{s}\right)$, and let $w \in W^{1, p}(\Omega)$. Assume that the sequence of norms $\left\|w_{s}\right\|_{W^{1, p}\left(\Omega_{s}\right)}$ is bounded and $\left\|w_{s}-q_{s} w\right\|_{L^{p}\left(\Omega_{s}\right)} \rightarrow 0$. Then $l_{s} w_{s} \rightarrow w$ weakly in $W^{1, p}(\Omega)$.

$\mathrm{Pr}$ o of. The properties of the operators $l_{s}$ imply that the sequence $\left\{l_{s} w_{s}\right\}$ is bounded in $W^{1, p}(\Omega)$ and

$$
\forall s \in \mathbb{N}, \quad q_{s}\left(l_{s} w_{s}\right)=w_{s} \text { a.e. in } \Omega_{s} .
$$

Assume that the sequence $\left\{l_{s} w_{s}\right\}$ does not converge weakly to $w$ in $W^{1, p}(\Omega)$. Then there exist a functional $g \in\left(W^{1, p}(\Omega)\right)^{*}$, a number $\varepsilon>0$, and an increasing sequence $\left\{\bar{s}_{k}\right\} \subset \mathbb{N}$ such that

$$
\forall k \in \mathbb{N}, \quad\left|\left\langle g, l_{\bar{s}_{k}} w_{\bar{s}_{k}}\right\rangle-\langle g, w\rangle\right|>\varepsilon .
$$

Since the space $W^{1, p}(\Omega)$ is reflexive and the sequence $\left\{l_{s} w_{s}\right\}$ is bounded in $W^{1, p}(\Omega)$, there exist an increasing sequence $\left\{s_{j}\right\} \subset\left\{\bar{s}_{k}\right\}$ and a function $w_{0} \in W^{1, p}(\Omega)$ such that

$$
l_{s_{j}} w_{s_{j}} \rightarrow w_{0} \text { weakly in } W^{1, p}(\Omega) .
$$

Hence, by condition $\left(*_{1}\right)$ of Theorem 1 , we have $l_{s_{j}} w_{s_{j}} \rightarrow w_{0}$ strongly in $L^{p}(\Omega)$. Then, in view of (17), we have $\left\|w_{s_{j}}-q_{s_{j}} w_{0}\right\|_{L^{p}\left(\Omega_{s_{j}}\right)} \rightarrow 0$. This and the assumption that $\left\|w_{s}-q_{s} w\right\|_{L^{p}\left(\Omega_{s}\right)} \rightarrow 0$ imply that $\left\|q_{s_{j}}\left(w-w_{0}\right)\right\|_{L^{p}\left(\Omega_{s_{j}}\right)} \rightarrow 0$. Consequently, $\liminf _{s \rightarrow \infty}\left\|q_{s}\left(w-w_{0}\right)\right\|_{L^{p}\left(\Omega_{s}\right)}=0$. From this equality, condition $\left(*^{\prime}\right)$ of Theorem 2 , and Proposition 3 , we derive that $w=w_{0}$ a.e. in $\Omega$. Then, in view of (19), we have $l_{s_{j}} w_{s_{j}} \rightarrow w$ weakly in $W^{1, p}(\Omega)$. However, this contradicts (18). The obtained contradiction proves that $l_{s} w_{s} \rightarrow w$ weakly in $W^{1, p}(\Omega)$.

The following definition essentially is a particular case of Definition 5 in [6].

Definition 6. Let, for every $s \in \mathbb{N}, U_{s}$ be a nonempty set in $W^{1, p}\left(\Omega_{s}\right)$, and let $U$ be a nonempty set in $W^{1, p}(\Omega)$. We say that the sequence $\left\{U_{s}\right\} \mathcal{H}$-converges to the set $U$ if the following conditions are satisfied:

(a) for every function $v \in U$, there exists a sequence $w_{s} \in U_{s}$ such that $\sup _{s \in \mathbb{N}}\left\|w_{s}\right\|_{W^{1, p}\left(\Omega_{s}\right)}<+\infty$ and $\left\|w_{s}-q_{s} v\right\|_{L^{p}\left(\Omega_{s}\right)} \rightarrow 0$

(b) for every sequence $v_{s} \in U_{s}$ such that $\sup _{s \in \mathbb{N}}\left\|v_{s}\right\|_{W^{1, p}\left(\Omega_{s}\right)}<+\infty$, there exist an increasing sequence $\left\{s_{j}\right\} \subset \mathbb{N}$ and a function $v \in U$ such that $\left\|v_{s_{j}}-q_{s_{j}} v\right\|_{L^{p}\left(\Omega_{s_{j}}\right)} \rightarrow 0$.

Proposition 6. Let condition $\left(*^{\prime}\right)$ of Theorem 2 be satisfied. Then a sequence of nonempty sets $U_{s} \subset W^{1, p}\left(\Omega_{s}\right)$ may $\mathcal{H}$-converge to only one nonempty set $U \subset W^{1, p}(\Omega)$.

$\mathrm{P}$ r o o f. Assume that a sequence of nonempty sets $U_{s} \subset W^{1, p}\left(\Omega_{s}\right) \mathcal{H}$-converges to nonempty sets $U \subset W^{1, p}(\Omega)$ and $V \subset W^{1, p}(\Omega)$. Let $w \in U$. Since the sequence $\left\{U_{s}\right\} \mathcal{H}$-converges to the set $U$, there exists a sequence $w_{s} \in U_{s}$ such that $\sup _{s \in \mathbb{N}}\left\|w_{s}\right\|_{W^{1, p}\left(\Omega_{s}\right)}<+\infty$ and $\left\|w_{s}-q_{s} w\right\|_{L^{p}\left(\Omega_{s}\right)} \rightarrow 0$. Since the sequence $\left\{U_{s}\right\} \mathcal{H}$-converges to the set $V$, for the sequence $\left\{w_{s}\right\}$, there exist an increasing sequence $\left\{s_{j}\right\} \subset \mathbb{N}$ and a function $v \in V$ such that $\left\|w_{s_{j}}-q_{s_{j}} v\right\|_{L^{p}\left(\Omega_{s_{j}}\right)} \rightarrow 0$. This convergence along with the convergence $\left\|w_{s}-q_{s} w\right\|_{L^{p}\left(\Omega_{s}\right)} \rightarrow 0$ implies that $\left\|q_{s_{j}}(v-w)\right\|_{L^{p}\left(\Omega_{s_{j}}\right)} \rightarrow 0$. Then, taking into account condition $\left(*^{\prime}\right)$ of Theorem 2 and Proposition 3, we find that $w=v$ a.e. in $\Omega$. Therefore, 
in view of the inclusion $v \in V$, we have $w \in V$. Consequently, $U \subset V$. In the same way, we prove that $V \subset U$. Thus, $U=V$.

Remark 1. In the proof of Proposition 6, concerning the considered sets in $W^{1, p}(\Omega)$, we implicitly assumed that functions equivalent to elements of these sets belong to the same sets.

Proposition 7. Assume that the embedding of $W^{1, p}(\Omega)$ into $L^{p}(\Omega)$ is compact and the sequence of spaces $W^{1, p}\left(\Omega_{s}\right)$ is strongly connected with the space $W^{1, p}(\Omega)$. Then the sequence $\left\{W^{1, p}\left(\Omega_{s}\right)\right\}$ $\mathcal{H}$-converges to the set $W^{1, p}(\Omega)$.

P r o o f. Let $v \in W^{1, p}(\Omega)$. For every $s \in \mathbb{N}$, we set $w_{s}=q_{s} v$. Obviously, for every $s \in \mathbb{N}$, we have $w_{s} \in W^{1, p}\left(\Omega_{s}\right)$. It is also easy to see that $\sup _{s \in \mathbb{N}}\left\|w_{s}\right\|_{W^{1, p}\left(\Omega_{s}\right)}<+\infty$ and $\left\|w_{s}-q_{s} v\right\|_{L^{p}\left(\Omega_{s}\right)} \rightarrow 0$. Next, taking a sequence $v_{s} \in W^{1, p}\left(\Omega_{s}\right)$ such that $\sup _{s \in \mathbb{N}}\left\|v_{s}\right\|_{W^{1, p}\left(\Omega_{s}\right)}<+\infty$, in view of the assumptions of this proposition, we deduce from Proposition 4 that there exist an increasing sequence $\left\{s_{j}\right\} \subset \mathbb{N}$ and a functon $v \in W^{1, p}(\Omega)$ such that $\left\|v_{s_{j}}-q_{s_{j}} v\right\|_{L^{p}\left(\Omega_{s_{j}}\right)} \rightarrow 0$. Now, by Definition 6, we conclude that the sequence $\left\{W^{1, p}\left(\Omega_{s}\right)\right\} \mathcal{H}$-converges to the set $W^{1, p}(\Omega)$.

We note that condition $\left(*^{\prime}\right)$ of Theorem 2 is essential for the conclusion of Proposition 6 . This is justified by the following simple example.

Example 3. Assume that $\Omega$ is a Lipschitz domain. Then the embedding of $W^{1, p}(\Omega)$ into $L^{p}(\Omega)$ is compact. Let $B$ be a closed ball in $\mathbb{R}^{n}$ such that $B \subset \Omega$, and assume that, for every $s \in \mathbb{N}, \Omega_{s}=\Omega \backslash B$. In view of the known extension results for Sobolev spaces (see, for instance, [25, Theorem 7.25]), there exists a linear continuous operator $l: W^{1, p}(\Omega \backslash B) \rightarrow W^{1, p}(\Omega)$ such that, for every function $v \in W^{1, p}(\Omega \backslash B)$, we have $l v=v$ in $\Omega \backslash B$. Setting, for every $s \in \mathbb{N}, l_{s}=l$, we find that the sequence $\left\{l_{s}\right\}$ has all the properties described in Definition 3. Therefore, the sequence of spaces $W^{1, p}\left(\Omega_{s}\right)$ is strongly connected with the space $W^{1, p}(\Omega)$. Thus, Proposition 7 implies that the sequence $\left\{W^{1, p}\left(\Omega_{s}\right)\right\} \mathcal{H}$-converges to the set $W^{1, p}(\Omega)$. Now, let $y$ and $r$ be the center and the radius of the ball $B$, respectively, and let $B_{0}=\left\{x \in \mathbb{R}^{n}:|x-y| \leqslant r / 2\right\}$. We define

$$
U=\left\{v \in W^{1, p}(\Omega): v=0 \text { a.e. in } B_{0}\right\} .
$$

It is easy to see that, for every function $v \in U$, there exists a sequence $w_{s} \in W^{1, p}\left(\Omega_{s}\right)$ such that $\sup _{s \in \mathbb{N}}\left\|w_{s}\right\|_{W^{1, p}\left(\Omega_{s}\right)}<+\infty$ and $\left\|w_{s}-q_{s} v\right\|_{L^{p}\left(\Omega_{s}\right)} \rightarrow 0$. Next, we fix an arbitrary sequence $v_{s} \in W^{1, p}\left(\Omega_{s}\right)$ such that $\sup _{s \in \mathbb{N}}\left\|v_{s}\right\|_{W^{1, p}\left(\Omega_{s}\right)}<+\infty$. Since the sequence $\left\{W^{1, p}\left(\Omega_{s}\right)\right\} \mathcal{H}$-converges to the set $W^{1, p}(\Omega)$, there exist an increasing sequence $\left\{s_{j}\right\} \subset \mathbb{N}$ and a function $v \in W^{1, p}(\Omega)$ such that

$$
\left\|v_{s_{j}}-q_{s_{j}} v\right\|_{L^{p}\left(\Omega_{s_{j}}\right)} \rightarrow 0 .
$$

Let $\varphi$ be a function in $C_{0}^{\infty}(\Omega)$ such that $0 \leqslant \varphi \leqslant 1$ in $\Omega, \varphi=1$ in $B_{0}$, and $\varphi=0$ in $\Omega \backslash B$. We have $v \varphi \in W^{1, p}(\Omega)$. Then, since $\varphi=1$ in $B_{0}$, we have $v-v \varphi \in U$. Moreover, taking into account that $\varphi=0$ in $\Omega \backslash B$, we derive from (20) that $\left\|v_{s_{j}}-q_{s_{j}}(v-v \varphi)\right\|_{L^{p}\left(\Omega_{s_{j}}\right)} \rightarrow 0$. Now, we conclude that the sequence $\left\{W^{1, p}\left(\Omega_{s}\right)\right\} \mathcal{H}$-converges to the set $U$. Obviously, $U \neq W^{1, p}(\Omega)$. It remains to observe that $\Omega \backslash \bigcup_{s=1}^{\infty} \Omega_{s}=B$. Hence, meas $\left(\Omega \backslash \bigcup_{s=1}^{\infty} \Omega_{s}\right)>0$. Consequently, condition $\left(*^{\prime}\right)$ of Theorem 2 is not satisfied.

We now proceed to a more delicate question on the $\mathcal{H}$-convergence of sets defined by bilateral constraints. 
Proposition 8. Assume that conditions $\left(*_{1}\right)$ and $\left(*_{2}\right)$ of Theorem 1 and condition $\left(*^{\prime}\right)$ of Theorem 2 are satisfied. Let $\varphi, \psi: \Omega \rightarrow \overline{\mathbb{R}}$, and let $\varphi \leqslant \psi$ a.e. in $\Omega$. Let, for every $s \in \mathbb{N}$, $U_{s}=\left\{v \in W^{1, p}\left(\Omega_{s}\right): \varphi \leqslant v \leqslant \psi\right.$ a.e. in $\left.\Omega_{s}\right\}$, and let $U=\left\{v \in W^{1, p}(\Omega): \varphi \leqslant v \leqslant \psi\right.$ a.e. in $\left.\Omega\right\}$. Assume that the set $U$ is nonempty. Then the sequence $\left\{U_{s}\right\} \mathcal{H}$-converges to the set $U$.

$\mathrm{P}$ r o o f. Let $v \in U$. For every $s \in \mathbb{N}$, we set $w_{s}=q_{s} v$. Obviously, for every $s \in \mathbb{N}$, we have $w_{s} \in U_{s}$. It is also easy to see that $\sup _{s \in \mathbb{N}}\left\|w_{s}\right\|_{W^{1, p}\left(\Omega_{s}\right)}<+\infty$ and $\left\|w_{s}-q_{s} v\right\|_{L^{p}\left(\Omega_{s}\right)} \rightarrow 0$.

Next, we fix an arbitrary sequence $v_{s} \in U_{s}$ such that $\sup _{s \in \mathbb{N}}\left\|v_{s}\right\|_{W^{1, p}\left(\Omega_{s}\right)}<+\infty$. Since condition $\left(*_{2}\right)$ of Theorem 1 is satisfied, there exists a sequence of linear continuous operators $l_{s}$ : $W^{1, p}\left(\Omega_{s}\right) \rightarrow W^{1, p}(\Omega)$ such that the sequence of norms $\left\|l_{s}\right\|$ is bounded and, for every $s \in \mathbb{N}$ and for every $v \in W^{1, p}\left(\Omega_{s}\right)$, we have $q_{s}\left(l_{s} v\right)=v$ a.e. in $\Omega_{s}$. Then, taking into account that condition $\left(*_{1}\right)$ of Theorem 1 is satisfied, we derive from Proposition 4 that there exist an increasing sequence $\left\{s_{j}\right\} \subset \mathbb{N}$ and a function $w \in W^{1, p}(\Omega)$ such that $l_{s_{j}} v_{s_{j}} \rightarrow w$ a.e. in $\Omega$ and $\left\|v_{s_{j}}-q_{s_{j}} w\right\|_{L^{p}\left(\Omega_{s_{j}}\right)} \rightarrow 0$. Let us show that $\varphi \leqslant w \leqslant \psi$ a.e. in $\Omega$. Since, for every $s \in \mathbb{N}$, we have $v_{s} \in U_{s}$, there exists a set $E^{\prime} \subset \Omega$ of measure zero such that, for every $s \in \mathbb{N}$ and for every $x \in \Omega_{s} \backslash E^{\prime}$, we have $\varphi(x) \leqslant v_{s}(x) \leqslant \psi(x)$. In addition, by the properties of the operators $l_{s}$, there exists a set $E^{\prime \prime} \subset \Omega$ of measure zero such that, for every $s \in \mathbb{N}$ and for every $x \in \Omega_{s} \backslash E^{\prime \prime}$, we have $\left(l_{s} v_{s}\right)(x)=v_{s}(x)$. It is clear that

$$
s \in \mathbb{N}, x \in \Omega_{s} \backslash\left(E^{\prime} \cup E^{\prime \prime}\right) \Longrightarrow \varphi(x) \leqslant\left(l_{s} v_{s}\right)(x) \leqslant \psi(x) .
$$

Since $l_{s_{j}} v_{s_{j}} \rightarrow w$ a.e. in $\Omega$, there exists a set $E^{\prime \prime \prime} \subset \Omega$ of measure zero such that

$$
\forall x \in \Omega \backslash E^{\prime \prime \prime}, \quad\left(l_{s_{j}} v_{s_{j}}\right)(x) \rightarrow w(x) .
$$

Next, for every $k \in \mathbb{N}$, we set $E^{(k)}=\Omega \backslash \bigcup_{j=k}^{\infty} \Omega_{s_{j}}$. In view of condition $\left(*^{\prime}\right)$ of Theorem 2 , for every $k \in \mathbb{N}$, we have meas $E^{(k)}=0$. Therefore, setting $E=\bigcup_{k=1}^{\infty} E^{(k)}$, we have meas $E=0$. Now, let $x \in \Omega \backslash\left(E^{\prime} \cup E^{\prime \prime} \cup E^{\prime \prime \prime} \cup E\right)$. We fix an arbitrary $\varepsilon>0$. Since $x \in \Omega \backslash E^{\prime \prime \prime}$, by (22), we have $\left(l_{s_{j}} v_{s_{j}}\right)(x) \rightarrow w(x)$. Consequently, there exists $k \in \mathbb{N}$ such that

$$
j \in \mathbb{N}, j \geqslant k \Longrightarrow\left|\left(l_{s_{j}} v_{s_{j}}\right)(x)-w(x)\right| \leqslant \varepsilon
$$

Since $x \in \Omega \backslash E$, there exists $j \in \mathbb{N}, j \geqslant k$, such that $x \in \Omega_{s_{j}}$. Then we derive from (21) and (23) that $\varphi(x)-\varepsilon \leqslant w(x) \leqslant \psi(x)+\varepsilon$. Hence, in view of the arbitrariness of $\varepsilon$, we obtain the inequality $\varphi(x) \leqslant w(x) \leqslant \psi(x)$. Therefore, $\varphi \leqslant w \leqslant \psi$ a.e. in $\Omega$. Then $w \in U$. Thus, we have established that, for every sequence $v_{s} \in U_{s}$ such that $\sup _{s \in \mathbb{N}}\left\|v_{s}\right\|_{W^{1, p}\left(\Omega_{s}\right)}<+\infty$, there exist an increasing sequence $\left\{s_{j}\right\} \subset \mathbb{N}$ and a function $w \in U$ such that $\left\|v_{s_{j}}-q_{s_{j}} w\right\|_{L^{p}\left(\Omega_{s_{j}}\right)} \rightarrow 0$.

We now conclude that the sequence $\left\{U_{s}\right\} \mathcal{H}$-converges to the set $U$.

We note that condition $\left(*^{\prime}\right)$ of Theorem 2 is essential for the conclusion of Proposition 8. This is justified by the following example.

Example 4. Assume that the domain $\Omega$ and the sequence of domains $\Omega_{s}$ are the same as in Example 3. Then conditions $\left(*_{1}\right)$ and $\left(*_{2}\right)$ of Theorem 1 are satisfied but condition $\left(*^{\prime}\right)$ of Theorem 2 is not satisfied. Let $\varphi: \Omega \rightarrow \overline{\mathbb{R}}$ be the function such that, for every $x \in \Omega, \varphi(x)=0$. Moreover, let $\psi: \Omega \rightarrow \overline{\mathbb{R}}$ be the function such that

$$
\psi(x)= \begin{cases}0 & \text { if } x \in B, \\ 1 & \text { if } x \in \Omega \backslash B .\end{cases}
$$


Obviously, $\varphi \leqslant \psi$ in $\Omega$. Let, for every $s \in \mathbb{N}, U_{s}=\left\{v \in W^{1, p}\left(\Omega_{s}\right): \varphi \leqslant v \leqslant \psi\right.$ a.e. in $\left.\Omega_{s}\right\}$, and let $U=\left\{v \in W^{1, p}(\Omega): \varphi \leqslant v \leqslant \psi\right.$ a.e. in $\left.\Omega\right\}$. Clearly, the set $U$ is nonempty. Thus, all the conditions of Proposition 8 are satisfied except for condition $\left(*^{\prime}\right)$ of Theorem 2 . At the same time, the sequence $\left\{U_{s}\right\}$ does not $\mathcal{H}$-converge to the set $U$. In fact, suppose that the sequence $\left\{U_{s}\right\}$ $\mathcal{H}$-converges to the set $U$. Then, taking the sequence $v_{s} \in W^{1, p}\left(\Omega_{s}\right)$ such that, for every $s \in \mathbb{N}$, $v_{s}=1$ in $\Omega_{s}$, we find that there exist an increasing sequence $\left\{s_{j}\right\} \subset \mathbb{N}$ and a function $v \in U$ such that $\left\|v_{s_{j}}-q_{s_{j}} v\right\|_{L^{p}\left(\Omega_{s_{j}}\right)} \rightarrow 0$. Hence, $v=1$ a.e. in $\Omega \backslash B$. Therefore, $v-1 \in \stackrel{\circ}{W^{1, p}}(\Omega)$. Moreover, since $v \in U$, we have $v=0$ a.e. in $B$. Thus, $|\nabla v|=0$ a.e. in $\Omega$. Then, fixing a number $r$ such that $1<r<\min \{p, n\}$ and taking into account that $v-1 \in \stackrel{\circ}{W}^{1, r}(\Omega)$, we apply the corresponding Sobolev inequality for the function $v-1$ and find that $v=1$ a.e. in $\Omega$. However, this contradicts the fact that $v=0$ a.e. in $B$. The obtained contradiction proves that the sequence $\left\{U_{s}\right\}$ does not $\mathcal{H}$-converge to the set $U$.

Although, in the general case, condition $\left(*^{\prime}\right)$ of Theorem 2 is essential for the $\mathcal{H}$-convergence of sets defined by bilateral constraints, in the case of regular constraints, this condition does not play any role for the $\mathcal{H}$-convergence of the corresponding sets. We demonstrate this by proving the following result.

Proposition 9. Assume that conditions $\left(*_{1}\right)$ and $\left(*_{2}\right)$ of Theorem 1 are satisfied. Let $\varphi, \psi \in$ $W^{1, p}(\Omega)$, and let $\varphi \leqslant \psi$ a.e. in $\Omega$. Let, for every $s \in \mathbb{N}, U_{s}=\left\{v \in W^{1, p}\left(\Omega_{s}\right): \varphi \leqslant v \leqslant \psi\right.$ a.e. in $\left.\Omega_{s}\right\}$, and let $U=\left\{v \in W^{1, p}(\Omega): \varphi \leqslant v \leqslant \psi\right.$ a.e. in $\left.\Omega\right\}$. Then the sequence $\left\{U_{s}\right\} \mathcal{H}$-converges to the set $U$.

P r o o f. As in the proof of Proposition 8, we establish that, for every function $v \in U$, there exists a sequence $w_{s} \in U_{s}$ such that $\sup _{s \in \mathbb{N}}\left\|w_{s}\right\|_{W^{1, p}\left(\Omega_{s}\right)}<+\infty$ and $\left\|w_{s}-q_{s} v\right\|_{L^{p}\left(\Omega_{s}\right)} \rightarrow 0$.

Next, we fix an arbitrary sequence $v_{s} \in U_{s}$ such that $\sup _{s \in \mathbb{N}}\left\|v_{s}\right\|_{W^{1, p}\left(\Omega_{s}\right)}<+\infty$. In view of condition $\left(*_{2}\right)$ of Theorem 1 , there exists a sequence of linear continuous operators $l_{s}: W^{1, p}\left(\Omega_{s}\right) \rightarrow$ $W^{1, p}(\Omega)$ such that the sequence of norms $\left\|l_{s}\right\|$ is bounded and

$$
\forall s \in \mathbb{N}, \quad q_{s}\left(l_{s} v_{s}\right)=v_{s} \text { a.e. in } \Omega_{s} .
$$

It is easy to see that the sequence $\left\{l_{s} v_{s}\right\}$ is bounded in $W^{1, p}(\Omega)$. For every $s \in \mathbb{N}$, we set

$$
z_{s}=\min \left\{\max \left\{l_{s} v_{s}, \varphi\right\}, \psi\right\} .
$$

We have $\left\{z_{s}\right\} \subset U$ and the sequence $\left\{z_{s}\right\}$ is bounded in $W^{1, p}(\Omega)$. Moreover, using (24) and the inclusions $v_{s} \in U_{s}$, we establish that

$$
\forall s \in \mathbb{N}, \quad q_{s} z_{s}=v_{s} \text { a.e. in } \Omega_{s} .
$$

Using the reflexivity of the space $W^{1, p}(\Omega)$, the boundedness of the sequence $\left\{z_{s}\right\}$ in $W^{1, p}(\Omega)$, and condition $\left(*_{1}\right)$ of Theorem 1 , we find that there exist an increasing sequence $\left\{s_{j}\right\} \subset \mathbb{N}$ and a function $v \in W^{1, p}(\Omega)$ such that

$$
z_{s_{j}} \rightarrow v \text { strongly in } L^{p}(\Omega)
$$

and $z_{s_{j}} \rightarrow v$ a.e. in $\Omega$. The latter limit relation along with the inclusion $\left\{z_{s_{j}}\right\} \subset U$ implies that $v \in U$. Finally, we derive from (25) and (26) that $\left\|v_{s_{j}}-q_{s_{j}} v\right\|_{L^{p}\left(\Omega_{s_{j}}\right)} \rightarrow 0$. Thus, we have established that, for every sequence $v_{s} \in U_{s}$ such that $\sup _{s \in \mathbb{N}}\left\|v_{s}\right\|_{W^{1, p}\left(\Omega_{s}\right)}<+\infty$, there exist an increasing sequence $\left\{s_{j}\right\} \subset \mathbb{N}$ and a function $v \in U$ such that $\left\|v_{s_{j}}-q_{s_{j}} v\right\|_{L^{p}\left(\Omega_{s_{j}}\right)} \rightarrow 0$. 
We now conclude that the sequence $\left\{U_{s}\right\} \mathcal{H}$-converges to the set $U$.

Remark 2. Concerning some notions of convergence of sets lying in the same space, see, for instance, $[26,27]$. Our notion of $\mathcal{H}$-convergence of sets lying generally in variable spaces differs from the notions of convergence of sets in the sense of Kuratowski [26, Section 29] and in the sense of Mosco [27, Definition 1.1] even in the case of sets belonging to the same space.

We give one more result involving condition $\left(*^{\prime}\right)$ of Theorem 2 .

Proposition 10. Let conditions $\left(*_{1}\right),\left(*_{2}\right),\left(*_{4}\right)$, and $\left(*_{5}\right)$ of Theorem 1 be satisfied. In addition, let condition $\left(*^{\prime}\right)$ of Theorem 2 be satisfied. Then there exist positive constants $b_{1}$ and $b_{2}$ such that, for every function $v \in W^{1, p}(\Omega)$, we have $(F+G)(v) \geqslant b_{1}\|v\|_{W^{1, p}(\Omega)}^{p}-b_{2}$.

P r o o f. By condition $\left(*_{2}\right)$ of Theorem 1, there exists a sequence of linear continuous operators $l_{s}: W^{1, p}\left(\Omega_{s}\right) \rightarrow W^{1, p}(\Omega)$ such that the sequence of norms $\left\|l_{s}\right\|$ is bounded and, for every $s \in \mathbb{N}$ and for every $v \in W^{1, p}\left(\Omega_{s}\right)$, we have $q_{s}\left(l_{s} v\right)=v$ a.e. in $\Omega_{s}$. We set $\lambda=\sup _{s \in \mathbb{N}}\left\|l_{s}\right\|$. It is not difficult to find that $\lambda$ is a real number such that $\lambda \geqslant 1$. Next, let $v \in W^{1, p}(\Omega)$. By virtue of condition $\left(*_{4}\right)$ of Theorem 1 , there exists a sequence $w_{s} \in W^{1, p}\left(\Omega_{s}\right)$ such that $\left\|w_{s}-q_{s} v\right\|_{L^{p}\left(\Omega_{s}\right)} \rightarrow 0$ and $F_{s}\left(w_{s}\right) \rightarrow F(v)$. The first of these limit relations and condition $\left(*_{5}\right)$ of Theorem 1 imply that $G_{s}\left(w_{s}\right) \rightarrow G(v)$. Thus,

$$
\left(F_{s}+G_{s}\right)\left(w_{s}\right) \rightarrow(F+G)(v) .
$$

In view of (7), we have

$$
\forall s \in \mathbb{N}, \quad\left(F_{s}+G_{s}\right)\left(w_{s}\right) \geqslant c_{5}\left\|w_{s}\right\|_{W^{1, p}\left(\Omega_{s}\right)}^{p}-c_{6} .
$$

This along with (27) implies that the sequence of norms $\left\|w_{s}\right\|_{W^{1, p}\left(\Omega_{s}\right)}$ is bounded. Now, since condition $\left(*_{1}\right)$ of Theorem 1 and condition $\left(*^{\prime}\right)$ of Theorem 2 are satisfied, we deduce from Proposition 5 that $l_{s} w_{s} \rightarrow v$ weakly in $W^{1, p}(\Omega)$. Therefore,

$$
\liminf _{s \rightarrow \infty}\left\|l_{s} w_{s}\right\|_{W^{1, p}(\Omega)} \geqslant\|v\|_{W^{1, p}(\Omega)} .
$$

Moreover, we have

$$
\forall s \in \mathbb{N}, \quad\left\|l_{s} w_{s}\right\|_{W^{1, p}(\Omega)} \leqslant \lambda\left\|w_{s}\right\|_{W^{1, p}\left(\Omega_{s}\right)} .
$$

From (27)-(30), we derive that $(F+G)(v) \geqslant c_{5} \lambda^{-p}\|v\|_{W^{1, p}(\Omega)}^{p}-c_{6}$.

We observe that condition $\left(*^{\prime}\right)$ of Theorem 2 is essential for the conclusion of Proposition 10. In this regard, see [10, Example 4.3].

We complete the exposition of the results related to condition $\left(*^{\prime}\right)$ of Theorem 2 with the following proposition.

Proposition 11. Assume that $c>0$ and, for every open set $H$ of $\mathbb{R}^{n}$ such that $H \subset \Omega$, we have $\liminf _{s \rightarrow \infty} \operatorname{meas}\left(H \cap \Omega_{s}\right) \geqslant c$ meas $H$. Then condition $\left(*^{\prime}\right)$ of Theorem 2 is satisfied.

Concerning the proof of this result, see, for instance, [10]. We also remark that the condition of Proposition 11 is satisfied in the case where the domains $\Omega_{s}$ have a perforated structure of the same kind as the structure of the domains considered in [16, Section 2].

Finally, we note that condition $\left(*^{\prime \prime}\right)$ of Theorem 2 is also important for the conclusion of this theorem. In this regard, see [10, Example 4.4]. Obviously, condition $\left(*^{\prime \prime}\right)$ of Theorem 2 is satisfied if all the functions $\mu_{s}$ are zero in the corresponding domains or if, for instance, for every $s \in \mathbb{N}$, we have $\mu_{s}=\left.\alpha_{s} \mu\right|_{\Omega_{s}}$, where $\left\{\alpha_{s}\right\} \subset[0,+\infty), \alpha_{s} \rightarrow 0$, and $\mu$ is a nonnegative function in $L^{1}(\Omega)$. 


\section{Conclusion}

In this paper, we have formulated and have discussed some results on the convergence of sequences of minimizers and minimum values of functionals $F_{s}+G_{s}: W^{1, p}\left(\Omega_{s}\right) \rightarrow \mathbb{R}$ on sets of functions defined by bilateral constraints in domains $\Omega_{s}$. These domains are assumed to be contained in a bounded domain $\Omega$ of $\mathbb{R}^{n}$. The functionals $F_{s}$ are integral and convex, and their integrands satisfy the bilateral estimate $c_{1}|\xi|^{p}-\mu_{s}(x) \leqslant f_{s}(x, \xi) \leqslant c_{2}|\xi|^{p}+\mu_{s}(x)$ for almost every $x \in \Omega_{s}$ and for every $\xi \in \mathbb{R}^{n}$, where $c_{1}$ and $c_{2}$ are positive constants and $\mu_{s}$ are nonnegative functions such that the sequence of norms $\left\|\mu_{s}\right\|_{L^{1}\left(\Omega_{s}\right)}$ is bounded. The functionals $G_{s}$ are assumed to be weakly continuous on the corresponding Sobolev spaces. They are generally not integral and play a subordinate role.

We have considered two cases: the case of regular constraints, i.e., constraints lying in the Sobolev space $W^{1, p}(\Omega)$, and the case where the lower constraint is zero and the upper constraint is an arbitrary nonnegative function. In both cases, a certain connection of the spaces $W^{1, p}\left(\Omega_{s}\right)$ with the space $W^{1, p}(\Omega)$, the $\Gamma$-convergence of the functionals $F_{s}$, and a convergence of the functionals $G_{s}$ are essentially used. At the same time, each of these cases has a distinctive feature. In the first case, it is required that the difference between the upper and lower constraints be positive almost everywhere. In the second case, this requirement is absent. However, in the latter case, it is assumed that $\left\|\mu_{s}\right\|_{L^{1}\left(\Omega_{s}\right)} \rightarrow 0$ and it is required that the exhaustion condition of the domain $\Omega$ by the domains $\Omega_{s}$ be satisfied.

We have given a series of results involving the exhaustion condition. In particular, we have obtained an equivalent statement of this condition and, using it, have proved the $\mathcal{H}$-convergence of sets of functions defined by bilateral (generally irregular) constraints in the domains $\Omega_{s}$.

\section{Acknowledgements}

This work was supported by the Program of the Ural Branch of the Russian Academy of Sciences "Current Problems in Algebra, Analysis, and the Theory of Dynamic Systems with Applications to the Control of Complex Objects" (project "Development of New Analytic, Numerical, and Asymptotic Methods for Problems of Mathematical Physics and Applications to Signal Processing") and by the Russian Academic Excellence Project (agreement no. 02.A03.21.0006 of August 27, 2013, between the Ministry of Education and Science of the Russian Federation and Ural Federal University).

\section{REFERENCES}

1. De Giorgi E., Franzoni T. Su un tipo di convergenza variazionale // Atti Accad. Naz. Lincei Rend. Cl. Sci. Fis. Mat. Natur. (8), 1975. Vol. 58, no. 6. P. 842-850.

2. Zhikov V.V. Questions of convergence, duality, and averaging for functionals of the calculus of variations // Math. USSR-Izv., 1984. Vol. 23, no. 2. P. 243-276. DOI: 10.1070/IM1984v023n02ABEH001466

3. Dal Maso G. An introduction to Г-convergence. Boston: Birkhäuser, 1993. 352 p. DOI: 10.1007/9781-4612-0327-8

4. Zhikov V.V. On passage to the limit in nonlinear variational problems // Russian Acad. Sci. Sb. Math., 1993. Vol. 76, no. 2. P. 427-459. DOI: 10.1070/SM1993v076n02ABEH003421

5. Kovalevskii A.A. Averaging of variable variational problems // Dokl. Akad. Nauk Ukr. SSR. Ser. A, 1988. No. 8. P. 6-9. [in Russian]

6. Kovalevskii A.A. On the connectedness of subsets of Sobolev spaces and the $\Gamma$-convergence of functionals with varying domain of definition // Nelinein. Granichnye Zadachi, 1989. Vol. 1. P. 48-54. [in Russian]

7. Kovalevskii A.A. On the $\Gamma$-convergence of integral functionals defined on Sobolev weakly connected spaces // Ukrainian Math. J., 1996. Vol. 48, no. 5. P. 683-698. DOI: 10.1007/BF02384235 
8. Hruslov E.Ya. The asymptotic behavior of solutions of the second boundary value problem under fragmentation of the boundary of the domain // Math. USSR-Sb., 1979. Vol. 35, no. 2. P. 266-282. DOI: 10.1070/SM1979v035n02ABEH001474

9. Kovalevsky A.A. On the convergence of solutions of variational problems with bilateral obstacles in variable domains // Proc. Steklov Inst. Math. (Suppl.), 2017. Vol. 296, suppl. 1. P. 151-163. DOI: 10.1134/S0081543817020146

10. Kovalevsky A.A. On the convergence of solutions to bilateral problems with the zero lower constraint and an arbitrary upper constraint in variable domains // Nonlinear Anal., 2016. Vol. 147. P. 63-79. DOI: 10.1016/j.na.2016.09.001

11. Vainberg M.M. Variational method and method of monotone operators in the theory of nonlinear equations. New York: Wiley, 1974. 356 p.

12. Kovalevskii A.A. Some problems connected with the problem of averaging variational problems for functionals with a variable domain // Current Analysis and its Applications. Kiev: Naukova dumka, 1989. P. $62-70$.

13. Kovalevsky A.A. Obstacle problems in variable domains // Complex Var. Elliptic Equ., 2011. Vol. 56, no. 12. P. 1071-1083. DOI: $10.1080 / 17476933.2010 .504842$

14. Adams R.A. Sobolev spaces. New York: Academic Press, 1975. 286 p.

15. Malý J., Ziemer W.P. Fine regularity of solutions of elliptic partial differential equations. Providence: AMS, 1997. 291 p.

16. Kovalevskii A.A. $G$-convergence and homogenization of nonlinear elliptic operators in divergence form with variable domain // Russian Acad. Sci. Izv. Math., 1995. Vol. 44, no. 3. P. 431-460. DOI: 10.1070/IM1995v044n03ABEH001607

17. Kovalevskij A.A. Conditions of the $\Gamma$-convergence and homogenization of integral functionals with different domains of the definition // Dokl. Akad. Nauk Ukr. SSR, 1991. No. 4. P. 5-8. [in Russian]

18. Kovalevskii A.A. On necessary and sufficient conditions for the $\Gamma$-convergence of integral functionals with different domains of definition // Nelinein. Granichnye Zadachi, 1992. Vol. 4. P. 29-39. [in Russian]

19. Kovalevskii A.A., Rudakova O.A. On the $\Gamma$-compactness of integral functionals with degenerate integrands // Nelinein. Granichnye Zadachi, 2005. Vol. 15. P. 149-145. [in Russian]

20. Rudakova O.A. On $\Gamma$-convergence of integral functionals defined on various weighted Sobolev spaces // Ukrainian Math. J., 2009. Vol. 61. P. 121-139. DOI: 10.1007/s11253-009-0193-1

21. Kovalevsky A.A. On $L^{1}$-functions with a very singular behaviour // Nonlinear Anal., 2013. Vol. 85. P. 66-77. DOI: 10.1016/j.na.2013.02.017

22. Murat F. Sur l'homogeneisation d'inequations elliptiques du 2ème ordre, relatives au convexe $K\left(\psi_{1}, \psi_{2}\right)=\left\{v \in H_{0}^{1}(\Omega) \mid \psi_{1} \leqslant v \leqslant \psi_{2}\right.$ p.p. dans $\left.\Omega\right\}$. 1976. Publ. Laboratoire d'Analyse Numérique, no. 76013. Univ. Paris VI. 23 p.

23. Kovalevsky A.A. Variational problems with unilateral pointwise functional constraints in variable domains // Trudy Inst. Mat. i Mekh. UrO RAN, 2017. Vol. 23, no. 2. P. 133-150. [in Russian] DOI: 10.21538/0134-4889-2017-23-2-133-150

24. Kovalevsky A.A., Rudakova O.A. Variational problems with pointwise constraints and degeneration in variable domains // Differ. Equ. Appl., 2009. Vol. 1, no. 4. P. 517-559. DOI: 10.7153/dea-01-29.

25. Gilbarg D., Trudinger N.S. Elliptic partial differential equations of second order. Berlin: Springer, 1983. 518 p. DOI: $10.1007 / 978-3-642-96379-7$

26. Kuratowski K. Topology. Vol. I. New York: Academic Press, 1966. 580 p.

27. Mosco U. Convergence of convex sets and of solutions of variational inequalities // Adv. Math., 1969. Vol. 3, no. 4. P. 510-585. 